Annales Geophysicae (2001) 19: 1-9 C) European Geophysical Society 2001

\title{
Dawn-dusk asymmetry in particles of solar wind origin within the magnetosphere
}

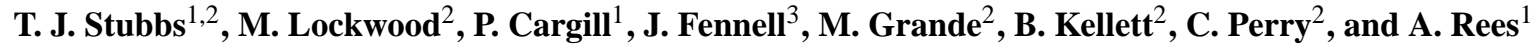 \\ ${ }^{1}$ Space \& Atmospheric Physics, The Blackett Laboratory, Imperial College, London, SW7 2BW, United Kingdom \\ ${ }^{2}$ Rutherford Appleton Laboratory, Chilton, Didcot, Oxfordshire, OX11 0QX, United Kingdom \\ ${ }^{3}$ The Aerospace Corporation, Mail Station M2-259, PO Box 92957, Los Angeles, CA 90009, U.S.A.
}

Received: 31 May 2000 - Revised: 20 October 2000 - Accepted: 30 October 2000

\begin{abstract}
Solar wind/magnetosheath plasma in the magnetosphere can be identified using a component that has a higher charge state, lower density and, at least soon after their entry into the magnetosphere, lower energy than plasma from a terrestrial source. We survey here observations taken over 3 years of $\mathrm{He}^{2+}$ ions made by the Magnetospheric Ion Composition Sensor (MICS) of the Charge and Mass Magnetospheric Ion Composition Experiment (CAMMICE) instrument aboard POLAR. The occurrence probability of these solar wind ions is then plotted as a function of Magnetic Local Time (MLT) and invariant latitude $(\Lambda)$ for various energy ranges. For all energies observed by MICS (1.8-21.4 keV) and all solar wind conditions, the occurrence probabilities peaked around the cusp region and along the dawn flank. The solar wind conditions were filtered to see if this dawnward asymmetry is controlled by the Svalgaard-Mansurov effect (and so depends on the $B_{Y}$ component of the interplanetary magnetic field, IMF) or by Fermi acceleration of $\mathrm{He}^{2+}$ at the bow shock (and so depends on the IMF ratio $B_{X} / B_{Y}$ ). It is shown that the asymmetry remained persistently on the dawn flank, suggesting it was not due to effects associated with direct entry into the magnetosphere. This asymmetry, with enhanced fluxes on the dawn flank, persisted for lower energy ions (below a "cross-over" energy of about $23 \mathrm{keV}$ ) but reversed sense to give higher fluxes on the dusk flank at higher energies. This can be explained by the competing effects of gradient/curvature drifts and the convection electric field on ions that are convecting sunward on re-closed field lines. The lower-energy $\mathrm{He}^{2+}$ ions $\boldsymbol{E} \times \boldsymbol{B}$ drift dawnwards as they move earthward, whereas the higher energy ions curvature/gradient drift towards dusk. The convection electric field in the tail is weaker for northward IMF. Ions then need less energy to drift to the dusk flank, so that the cross-over energy, at which the asymmetry changes sense, is reduced.
\end{abstract}

Key words. Magnetospheric physics (magnetospheric configuration and dynamics; magnetopause, cusp, and boundary

Correspondence to: T. J. Stubbs (tj.stubbs@ic.ac.uk) layers) - Space plasma physics (charged particle motion and acceleration)

\section{Introduction}

The mechanisms by which solar wind ions are energised and transported in the geospace environment rely on many timedependent parameters and, as a result, are complicated and not well understood. In the terrestrial magnetosphere, there is strong evidence showing that solar wind plasma gains access to the magnetosphere by flowing along open magnetic field lines from the magnetosheath. These open field lines are produced by magnetic reconnection taking place at the dayside magnetopause which maps to invariant latitudes ranging from about $75^{\circ}$ to $85^{\circ}$. This study examines the region with invariant latitudes greater than $60^{\circ}$ containing open field line features such as the cusp and polar cap as well as the open/ closed field line boundary, the low-latitude boundary layer (LLBL - whether on open or closed field lines) and the auroral oval on closed field lines. In this region we can infer the nature of global processes of ion injection and transport from the energy and distribution of solar wind ions. In the magnetosphere solar wind plasma is characterised by a component of the ion gas with higher charge state (for example, $\mathrm{He}^{2+}$ and $\left.\mathrm{O}^{>2+}\right)$, lower energy $(\sim 1 \mathrm{keV})$ and lower density relative to other populations in the magnetosphere. However, as they are transported around the magnetosphere by convection, solar wind ions become energised and therefore can no longer be differentiated by their energy. However, charge exchange takes place only very slowly and therefore the charge state can be used to detect their origin in the solar wind.

Sometimes the POLAR spacecraft has observed intense fluxes of solar wind ions in and around the cusp for prolonged periods. These are known as extended cusp-like regions (ECRs) and are sometimes observed during persistent, strong northward IMF $B_{Z}$. A study by Chen et al. (1998) discussed observations of $\mathrm{He}^{2+}$ ions lasting for a few hours with energies from 1-200 keV/e in the dayside cusp around 
local noon, with a $79 \%$ bias to the afternoon side. On the 29 May 1996 an ECR event, characterised by high fluxes of solar wind ions over a large range of invariant latitudes and magnetic local times, coincided with a persistent, strong northward IMF. This, along with other evidence, lead Grande et al. (1997) and Chandler et al. (1999) to the conclusion that this particular ECR was caused by magnetic reconnection in the tail-lobe. For this same event, Onsager et al. (2000) used electron fluxes to show that POLAR had crossed into the magnetosheath. Initial studies showed no direct correlation between ECRs and northward IMF, nor in fact any other solar wind parameter (Stubbs et al., 2000). However, ions that we can identify as originating from the solar wind are not just found in ECRs. Some cusp crossings last just a few seconds (implying narrow latitudinal width) and solar wind ions are also seen in the low-latitude boundary layer (LLBL), the mantle, the tail-lobe and in the plasma sheet (see Newell and Meng, 1992). Perry et al. (2000) have shown that high charge state ion abundances in ECRs follow these seen at the L1 point in the solar wind, both varying with the temperature of the source region in the solar corona.

In this paper, we use occurrence probabilities to analyse the distribution of solar wind ions in the magnetosphere using $\mathrm{He}^{2+}$ as a tracer. Section 2 gives details of the two spacecraft and three instruments used in this study. Particular attention is paid to the CAMMICE MICS ion detector aboard POLAR which has been the focus of this work. The method used to process and plot the data in terms of occurrence probabilities is also explained. Section 3 presents the results and interpretation of the occurrence probability distributions.

\section{Spacecraft and instrumentation}

The data for this study has come from the two Global Geospace Science (GGS) spacecraft, POLAR and WIND. POLAR is in a $1.8 \times 9 R_{E}$ polar orbit with an inclination of $\sim 86^{\circ}$ to the equator and a period of $\sim 17.5$ hours (Acuña et al., 1995). Its apogee is at high northern latitudes and is spinstabilised at $10 \mathrm{rpm}$ with its spin axis approximately perpendicular to the orbital plane. The data set used in this study is from the Magnetospheric Ion Composition Sensor (MICS) in the Charge and Mass Magnetospheric Ion Composition Experiment (CAMMICE). The Electrostatic Analyser (ESA) on MICS filters by energy per charge $(E / q)$ and has 32 steps (0-31) with $E / q$ mid-points ranging from 1.0-416.7 keV/e. The ESA has a $\Delta E / E \sim 16 \%$ full width at half maximum, so the $E / q$ range of the detector is effectively 0.9 to 450.0 $\mathrm{keV} / \mathrm{e}$. For most of the mission, only steps 0-24 have been used, reducing the effective energy range to $0.9-208.8 \mathrm{keV} / \mathrm{e}$. Filtering by the ESA, combined with time-of-flight measurements and determination of the energy deposited in the solid state detector, allows MICS to determine the flux, mass and charge states of the incident ions (Wilken et al., 1992). In this study we are interested in lower energy $\mathrm{He}^{2+}$ ions, such as those found within the cusp and LLBL, and thus restricted the survey to consider ion energies covered by steps $0-9$ giv- ing a range of $0.9-10.7 \mathrm{keV} / \mathrm{e}$. The data for this study was taken between 18 March 1996 and 13 April 1999, giving over three years of almost continuous coverage.

To relate CAMMICE observations to solar wind conditions we use data from the WIND spacecraft. The orbit of the WIND spacecraft in this interval takes it from the nightside magnetosphere to the L1 libration point, though it spends most of its time in the solar wind upstream of the Earth. Data was used from the MFI (Magnetic Fields Investigation) and the SWE (Solar Wind Experiment) (respectively, Lepping et al., 1995 and Ogilvie et al., 1995) obtained from NSSDC CDAWeb. The MFI and SWE data sets were merged and averaged over 5 minutes. To calculate the convection time from WIND to the Earth in the GSM X-direction, we simply divided the distance from WIND to the centre of the Earth by the velocity of the solar wind, $V_{s w}$. This assumes that the structure and properties of the solar wind do not vary greatly over distances of $\sim 100 R_{E}$ transverse to the Sun-Earth line, appropriate for well organised events, and that the bulk of the motion is along the Sun-Earth line. It also assumes that the propagation time across the subsolar magnetosheath is approximately equal to $X_{m} / V_{s w}$, where $X_{m}$ is the stand-off distance of the magnetopause.

A list of $197.5 \mathrm{~s}$ integration periods when MICS observed $\mathrm{He}^{2+}$ was generated, along with the peak count rate and the ESA step in which the peak rate occurred. This was assumed to point to the average energy of the $\mathrm{He}^{2+}$ ions being observed. The raw counts were divided by the detector efficiency for $\mathrm{He}^{2+}$ at each ESA step, with the detection efficiency being higher for higher ion energies. If this was not done, the lower ESA steps would be underrepresented. MICS takes data at one ESA step per rotation (6 s), so it would need $192 \mathrm{~s}$ to make an observation at each ESA step, plus $5.5 \mathrm{~s}$ to reset for the next set of measurements. Thus, raw count rates $\left(\mathrm{s}^{-1}\right)$ are calculated by averaging over $6 \mathrm{~s}$. To avoid using single counts, which can be created by anomalous events in the detectors, we set the minimum raw count rate at $1 \mathrm{~s}^{-1}$ and so 6 counts in an integration period were required for it to register $\mathrm{He}^{2+}$ as present. A maximum raw count rate of $30 \mathrm{~s}^{-1}$ was also set, as cosmic rays striking a detector can lead to anomalously high count rates $(\sim 1000$ $\mathrm{s}^{-1}$ ).

From the list of MICS $\mathrm{He}^{2+}$ observations and the 5-min averaged solar wind parameters from WIND, we plotted the occurrence probability of observing $\mathrm{He}^{2+}$ as a function of invariant latitude and magnetic local time (MLT). These plots cover invariant latitudes from $60^{\circ}$ to $90^{\circ}$ at all magnetic local times in $1.5^{\circ}$ by 24 min bins, as shown in Fig. 1. The occurrence probability was calculated by dividing the number of times POLAR observed $\mathrm{He}^{2+}$ by the total number of samples made in that bin for the conditions in question. Plotting occurrence probabilities removes any biasing in the data caused by the orbit or instrument sampling. To ensure all bins are statistically significant, we have removed any bins sampled less than 15 times. This means an anomalous event will only register a $7 \%$ probability at most. The occurrence probability scale bar was standardised from 0 to $30 \%$ in the 

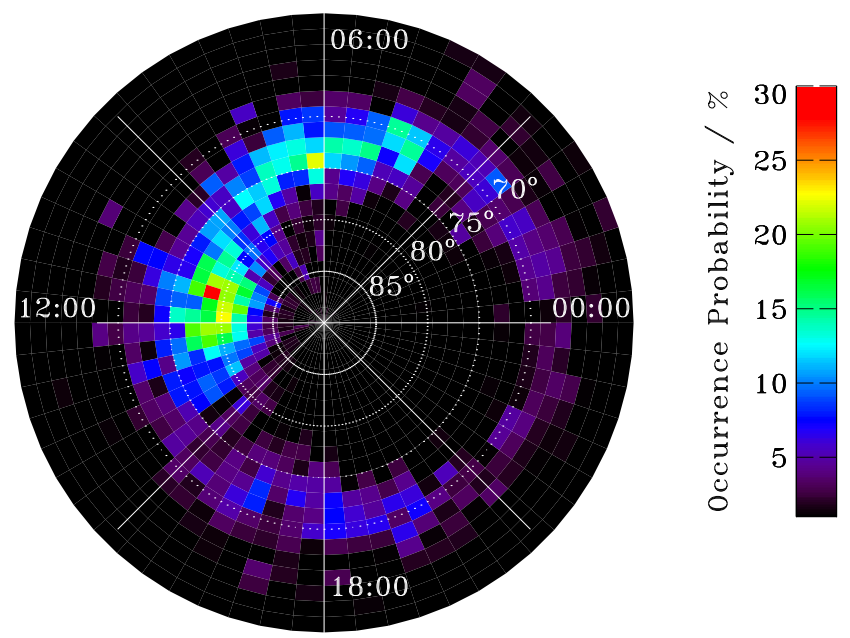

Fig. 1. The occurrence probability distribution of $\mathrm{He}^{2+}$ ions in the magnetosphere plotted as invariant latitude $(\Lambda)$ versus magnetic local time (MLT) in polar co-ordinates. In addition to the cusp near noon, an interesting feature in this plot is the peak in the occurrence probabilities along the dawn flank, $\Lambda \sim 72^{\circ}$. The plot is for $\Lambda$ from $60^{\circ}$ to $90^{\circ}$ for all MLTs with a bin size of $1.5^{\circ}$ by $24 \mathrm{~min}$. The energy of $\mathrm{He}^{2+}$ ions ranges from 1.8 to $21.4 \mathrm{keV}$ and samples are for all orientations of the IMF. Each bin has a 15 sample threshold to ensure reliable statistics. CAMMICE MICS data used in this plot was taken almost continuously from 18 March 1996 to 13 April 1999.

plots to allow direct comparison.

Figure 1 shows the distribution for $\mathrm{He}^{2+}$ ions with energies from 1.8 to $21.4 \mathrm{keV}$ for all interplanetary conditions. This plot shows a peak in the distributions along the dawn flank. Section 3 is divided into three sub-sections where possible mechanisms that could give rise to this dawn-dusk asymmetry are tested and discussed. Sub-section 3.1 tests the Svalgaard- Mansurov effect by sorting the samples by the direction of the IMF $B_{Y}$ component. Similarly, sub-section 3.2 tests the Fermi acceleration of ions at the bow shock by sorting the sample by the sense of the ratio IMF $B_{X} / B_{Y}$, i.e. sorting by Parker spiral and ortho-Parker spiral. Finally, in sub-section 3.3 the data is sorted by $\mathrm{He}^{2+}$ ion energy and the sense of the IMF $B_{Z}$ component. This tests for the effects of drift shells on the $\mathrm{He}^{2+}$ population and some other suggested mechanisms. In Sect. 4, a summary of results is given and placed in the context of the global magnetosphere.

\section{Results and interpretation}

Figure 1 shows the occurrence probabilities for $\mathrm{He}^{2+}$ ions from $1.8-21.4 \mathrm{keV}$ under all interplanetary conditions. The cusp is prominent near local noon, centred at about $79^{\circ}$ invariant latitude; the low-latitude boundary layer (LLBL) is shown ranging from about $68^{\circ}$ to about $75^{\circ}$ invariant latitude and extends over a large range of local times. This appears to be contiguous with a band that covers all local times, but with a distinct asymmetry, $\mathrm{He}^{2+}$ being seen most often on

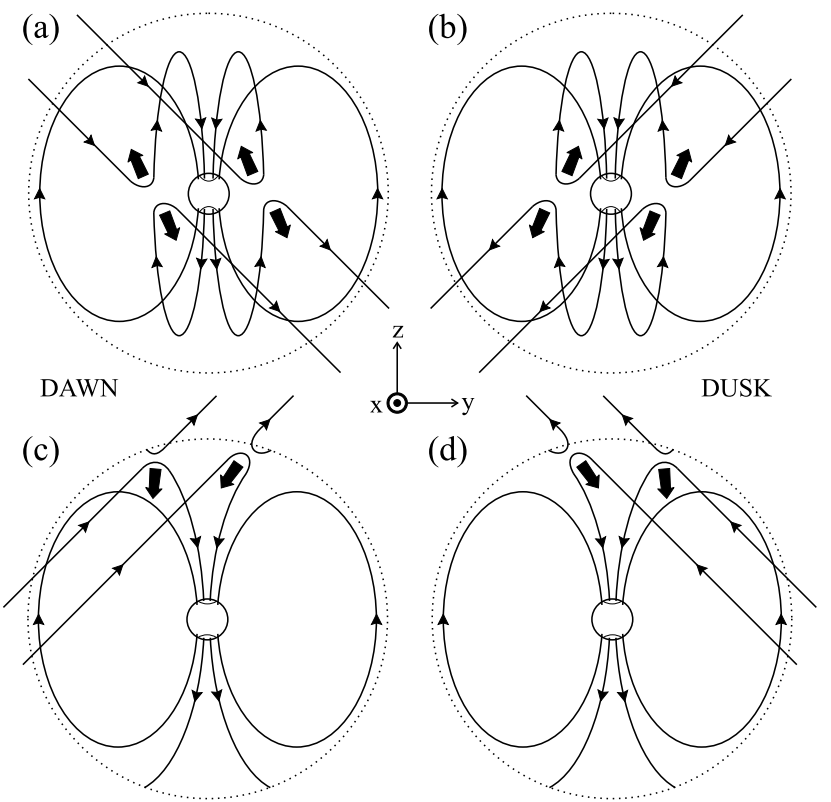

Fig. 2. The asymmetry in the evolution of newly-opened field lines and its dependence on the orientation of IMF $B_{Y}$ for southward IMF ( $\mathbf{a}$ and $\mathbf{b}$ ), as described by the Svalgaard-Mansurov effect (after Gosling et al., 1990). The asymmetry in the evolution of newlyopened field lines for northward IMF is similarly shown (c and d) where reconnection is taking place in the northern hemisphere taillobe. Solid lines are magnetic field, dotted lines are the magnetopause boundary and the large arrows show the direction of curvature force. Shown in GSM co-ordinates.

the dawn flank and least often in the pre-midnight sector. We explored three potential causes for the asymmetry:

1. The Svalgaard-Mansurov effect and its dependence on IMF $B_{Y}$

2. Fermi acceleration at the bow shock, dependent on the ratio IMF $B_{X} / B_{Y}$

3. Drift shells dependent on convection electric fields in the tail.

Both the Svalgaard-Mansurov effect and Fermi acceleration at the bow shock would result in an asymmetry of solar wind ions, but this asymmetry would be on open field lines where solar wind ions gain direct access to the magnetosphere. Drift shells are on closed field lines, which were once open but have been closed by reconnection in the central current sheet of the tail. Thus, in all three cases, the ions gain entry along open field lines. However, the dawn-dusk asymmetry has different causes. Another mechanism for creating an asymmetry in the distribution of magnetosheath ions was proposed by Olson and Pfitzer (1985). They suggested that magnetosheath ions could gradient drift onto closed magnetospheric field lines. This mechanism would cause ions to enter on the dawn flank, where higher energy ions would enter more readily due to their greater gyro-radii. 

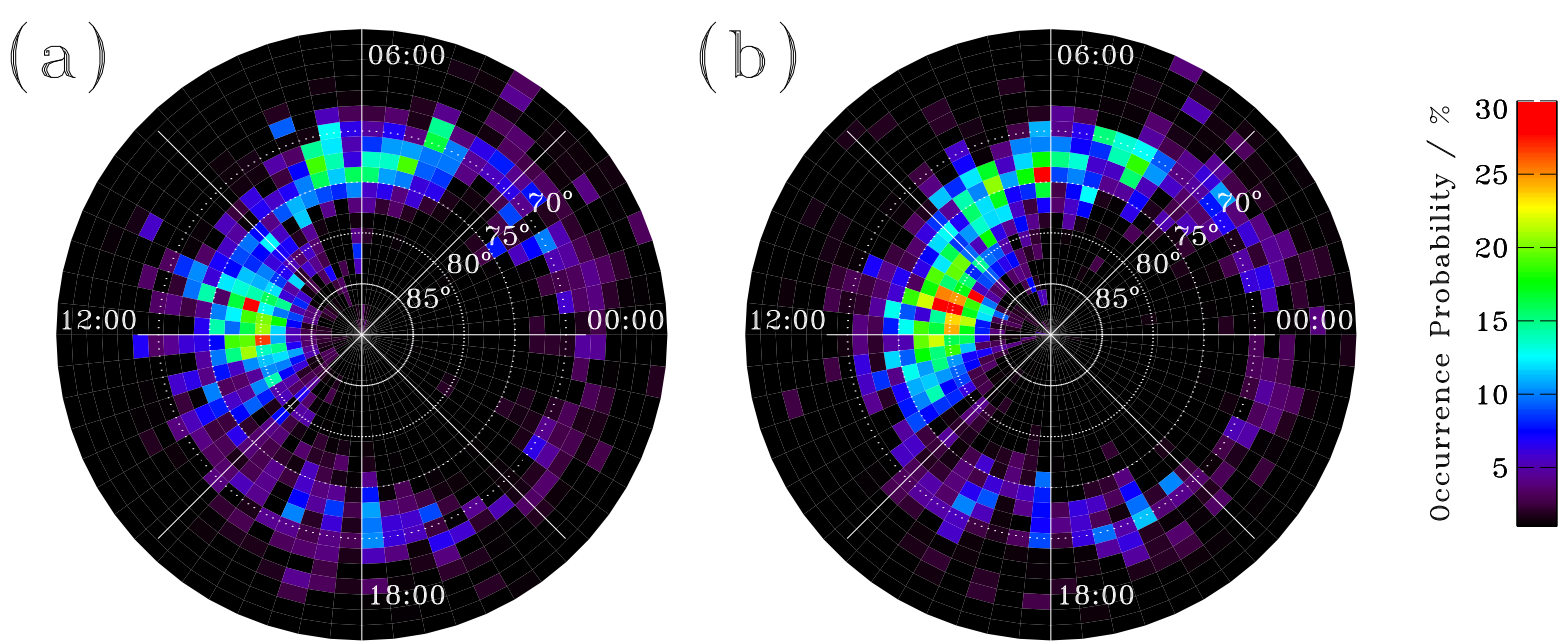

Fig. 3. $\mathrm{He}^{2+}$ ion occurrence probabilities sampled during (a) IMF $B_{Y}>0$ and (b) IMF $B_{Y}<0$ conditions. The data are shown in the same format as Fig. 1.

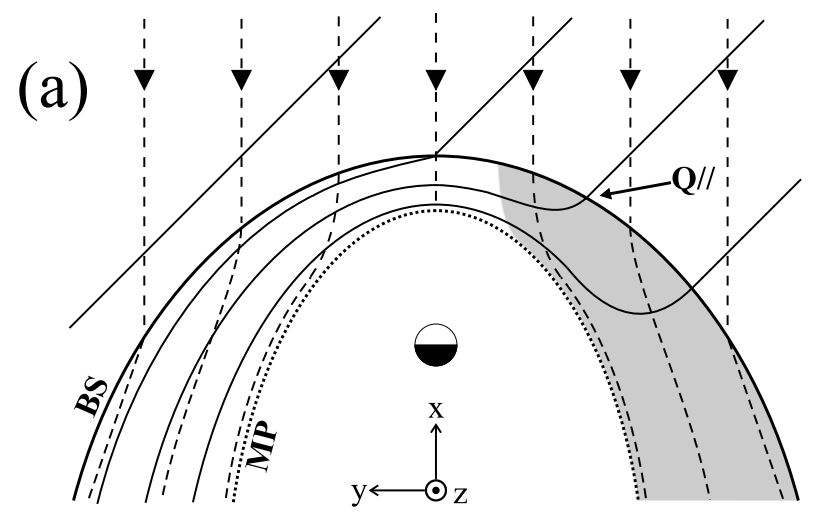

\subsection{Svalgaard-Mansurov effect}

The Svalgaard-Mansurov effect occurs when the IMF $B_{Y^{-}}$ component creates an asymmetry about the noon-midnight meridian in the flow of newly- reconnected flux tubes as they move away from an $X$-line on the dayside magnetopause (Fig. 2). The geomagnetic field at the sub-solar point is close to being aligned with the GSM positive $Z$-direction, so reconnection with a draped IMF having a GSM $Y$-component results in reconnected field lines with a curvature such that the curvature force has a component in both the $Z$ and $Y$ directions when sub-solar reconnection occurs. For sub-solar reconnection, the flux tubes flow dawnward for $B_{Y}>0$ (Fig.

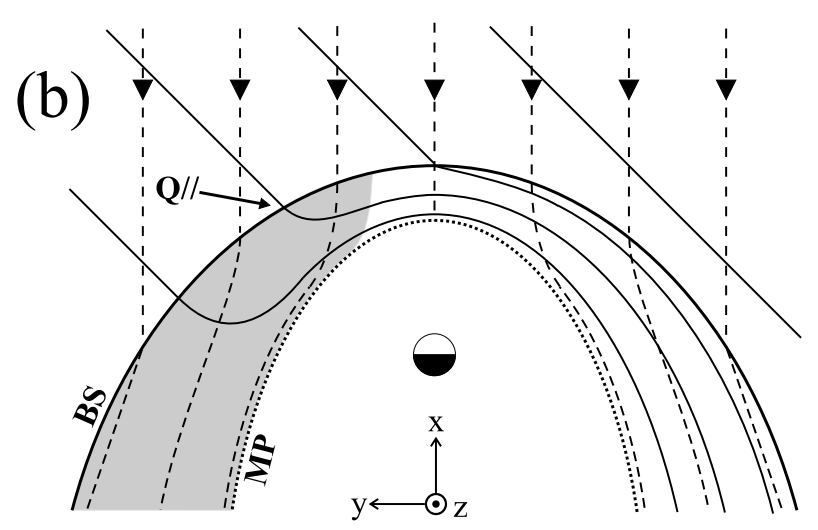
2a) and duskward for $B_{Y}<0$ (Fig. 2b) in the northern hemisphere. The asymmetry is reversed in the southern hemisphere. The sense of $B_{Y}$ leads to the same flow asymmetry for both IMF $B_{Z}<0$ and $B_{Z}>0$ (see Figs. 2c, d). This effect was first observed in the associated ionospheric Hall currents (e.g. Friis-Christensen et al., 1985) and later supported by low-altitude spacecraft observations (Heelis et al., 1984), radar data (Greenwald et al., 1990) and spacecraft observations at the magnetopause (Gosling et al., 1990).

To establish if the Svalgaard-Mansurov effect was responsible, we sorted the data into two subsets with IMF $B_{Y}>0$ and $B_{Y}<0$, the occurrence probability plots for which are shown in Figs. 3a, b, respectively. If the asymmetric evolution of newly-reconnected field lines was the explanation of the asymmetry, in the northern hemisphere it would favour high $\mathrm{He}^{2+}$ occurrence at the dawn flank for $B_{Y}>0$, but this would change to the dusk flank for $B_{Y}<0$. Figures 3a, $\mathrm{b}$ show that this does not occur, and therefore, we can conclude that the Svalgaard-Mansurov effect is not responsible for the asymmetry.

\subsection{Shock acceleration}

Ions can undergo Fermi acceleration at the bow shock where a quasi-parallel shock is formed, i.e. the IMF field lines are 

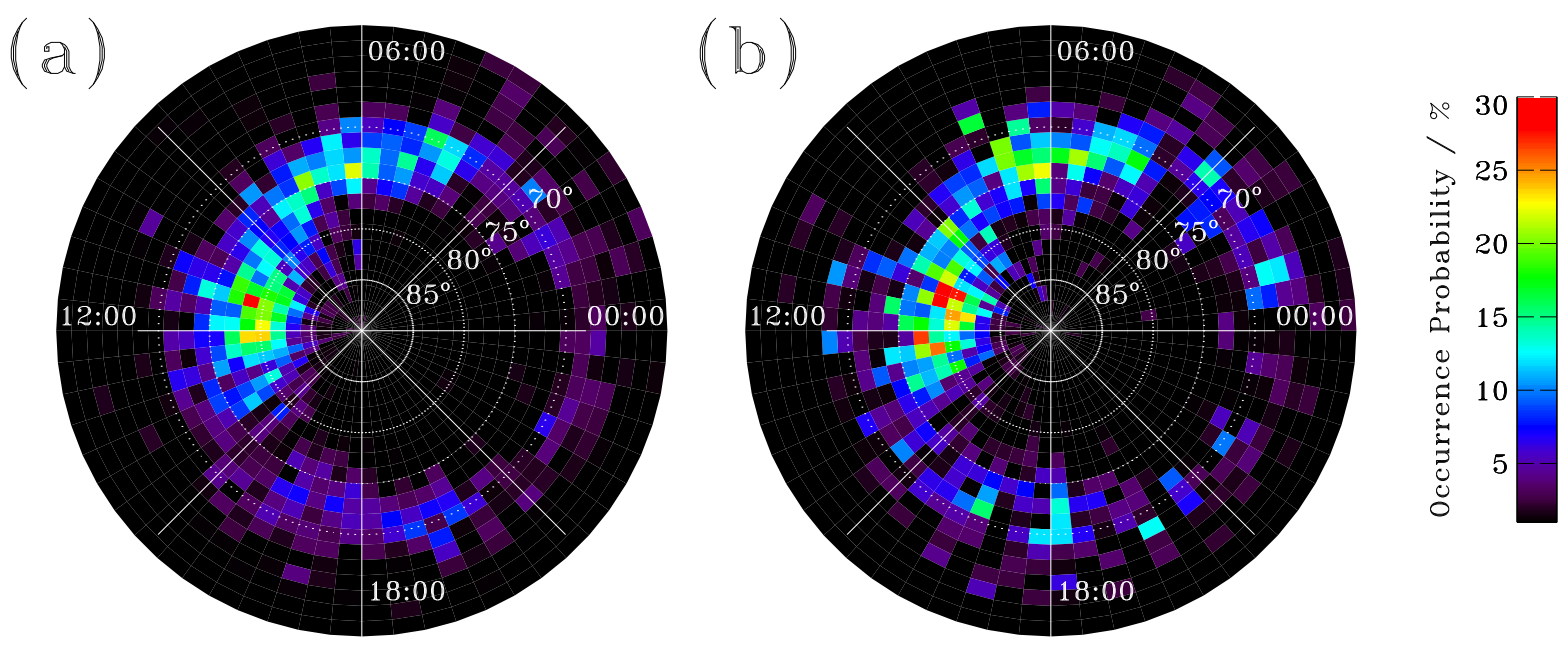

Fig. 5. $\mathrm{He} \mathrm{e}^{2+}$ ion occurrence probabilities sampled during (a) IMF $B_{X} / B_{Y}<0$ and (b) IMF $B_{X} / B_{Y}>0$ conditions. The data are shown in the same format as Fig. 1 .

parallel to the normal of the bow shock (Blandford and Eichler, 1987; Fuselier et al., 1994, 1997, 1998). The orientation of the Parker spiral (i.e. the sense of $B_{X} / B_{Y}$ ) dictates on which side of the bow shock this acceleration process can take place, as shown in Fig. 4. It makes no difference whether the IMF is pointing towards or away from the shock normal, the basic acceleration process is the same.

The data were sorted into subsets with $B_{X} / B_{Y}<0$ (Parker spiral) and $B_{X} / B_{Y}>0$ (ortho-Parker spiral). For a Parker spiral IMF we would expect shock accelerated solar wind ions to be formed on the dawn flank (Fig. 4a), while for ortho-Parker spiral we would expect them on the dusk flank (Fig. 4b). On average the IMF spends $\sim 75 \%$ of the time in the Parker spiral IMF configuration and the remaining 25\% in the ortho-Parker spiral configuration (e.g. Hapgood et al., 1991). Thus, the overall occurrence frequency of these two situations could explain the asymmetry in Fig. 1. However, from Figs. 5a, b we can see that the asymmetry persists on the dawn flank for both Parker spiral and ortho-Parker spiral orientations. Therefore, Fermi acceleration of $\mathrm{He}^{2+}$ ions at the bow shock is not the responsible mechanism.

\subsection{Drift shells}

If $\mathrm{He}^{2+}$ ions were to be carried to the dawn flank on closed field lines from the magnetotail, then a third cause of the asymmetry is possible. After solar wind ions enter the magnetosphere on open field lines, they get carried anti-sunward with convecting open field lines. The slower ions in the distribution can remain sunward of the reconnection site in the time taken for the field line is closed again. Therefore, instead of being returned to the solar wind, these ions remain in the magnetosphere on newly closed field lines that convect Earthward from the tail $X$-line. As these ions convect Earthward, they are accelerated by the curvature force acting on the field lines and are energised each time that they cross the tail current sheet (Onsager et al., 1991). Low-energy ions will tend to move toward the dawn flank of the magnetosphere under the influence of electric fields (close to earth, this is the sum of the convection electric field driven by the solar wind flow and the co-rotation electric field driven by the rotation of Earth's ionosphere). High energy ions will tend to move towards dusk under the gradient and curvature drifts (DeForest and McIlwain, 1971).

To investigate this possibility, we sorted the data by IMF $B_{Z}$, and the average $\mathrm{He}^{2+}$ ion energy observed by MICS. To improve the statistical significance of these plots, the $E / q$ mid-point range was extended to $1.0-23.3 \mathrm{keV} / \mathrm{e}$, giving an energy range from $1.8-50.3 \mathrm{keV}$.

An examination of energies less than $6.9 \mathrm{keV}$ (Figs. 6a, b) isolates the cusp around noon. For IMF $B_{Z}<0$ the cusp has a broad range in local time and is seen from about $72^{\circ}$ to about $77^{\circ}$ invariant latitude as shown in Fig. 6b. For IMF $B_{Z}>0$, the cusp is narrower in local time and is seen from about $78^{\circ}$ to about $83^{\circ}$ (see Fig. 6a). The more equatorward position of the cusp during southward IMF (Fig. 6b) than during northward IMF (Fig. 6a) shown here is seen in previous surveys of cusp precipitation (e.g. Burch, 1973; Newell et al., 1989) and in $630 \mathrm{~nm}$ cusp aurora (Horwitz and Akasofu, 1977; Sandholt et al., 1999) and is expected as the low-altitude signature of magnetopause erosion by magnetic reconnection during southward IMF.

For intermediate energies from 7.7 to $21.4 \mathrm{keV}$, the asymmetric feature on the dawn flank is isolated. This is shown for IMF $B_{Z}>0$ (northward) in Fig. $6 c$ and for IMF $B_{Z}<$ 0 in Fig. $6 d$ and demonstrates that the asymmetry on the dawn flank of the low latitude boundary layer/auroral oval is much stronger for southward IMF, whereas for northward IMF (Fig. 6c) an appreciable (but still smaller) peak in $\mathrm{He}^{2+}$ occurrence is forming near dusk.

Examining energies between 24.3 and $50.3 \mathrm{keV}$, we see that the asymmetry changes from the dawn to the dusk flank. 

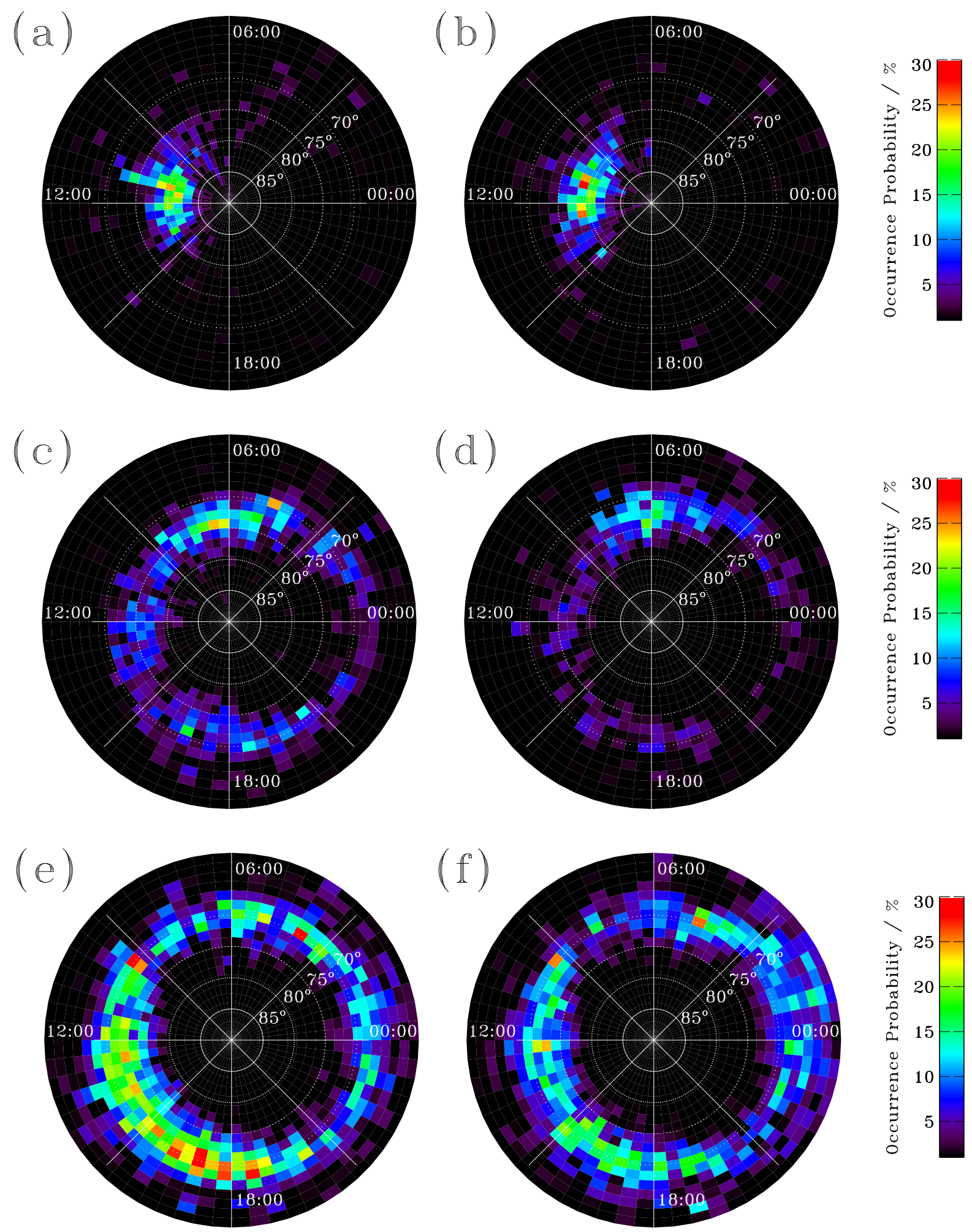

Fig. 6. $\mathrm{He}{ }^{2+}$ ion occurrence probabilities sampled during (a) IMF $B_{Z}>0$ and (b) IMF $B_{Z}<0$ conditions, for energies between 1.8 and $6.9 \mathrm{keV}$. Probabilities sampled during (c) IMF $B_{Z}>0$ and (d) IMF $B_{Z}<0$ conditions for energies between 7.7 and $21.4 \mathrm{keV}$. Probabilities sampled during (e) IMF $B_{Z}>0$ and (f) IMF $B_{Z}<0$ conditions for energies between 24.3 and $50.3 \mathrm{keV}$. The data are shown in the same format as Fig. 1. 
The extent of this change is dependent upon the sense of the IMF $B_{Z}$. For IMF $B_{Z}>0$ (Fig. 6e), the dusk flank bias is stronger than that observed for IMF $B_{Z}<0$ (Fig. 6f).

The gross electric field in the inner magnetosphere is a superposition of the electric field due to the co-rotation of the ionosphere with the Earth and the convection electric field associated with the earthward convection from the $X$-line in the tail. The co-rotation electric field varies diurnally, but is fairly constant. By comparison, the electric field in the tail is strongly dependent upon the state of global magnetospheric convection and so the rate of reconnection and the orientation of IMF $B_{Z}$. Convection is strongest during southward IMF and, as a result, so is the associated electric field. As ions approach the Earth from the tail, they come under the influence of opposing forces: the $\boldsymbol{E} \times \boldsymbol{B}$ force drifting the ions to the dawn flank and the opposing gradient $B$ and curvature forces which drift the ions to the dusk flank. The relative strength of these forces determines the path of ions and the nature of the dawn-dusk asymmetry (DeForest and McIlwain, 1971). An important difference between these two forces is that the $\boldsymbol{E} \times \boldsymbol{B}$ depends only on the local electric and magnetic field strengths (see Eq. 1); whereas both the gradient and curvature drifts (see Eqs. 2 and 3 respectively) are dependent upon ion energy.

$$
\begin{aligned}
v_{E} & =\frac{\boldsymbol{E} \times \boldsymbol{B}}{B^{2}} \\
v_{\nabla} & =\frac{m v_{\perp}^{2}}{2 q B^{3}}(\boldsymbol{B} \times \nabla B) \\
v_{R} & =\frac{m v_{\|}^{2}}{q R_{C}^{2} B^{2}}\left(\boldsymbol{R}_{C} \times \boldsymbol{B}\right)
\end{aligned}
$$

As a result, one would expect the $\boldsymbol{E} \times \boldsymbol{B}$ force to have a stronger influence during southward IMF than northward IMF. This effect can be seen in Figs. 6e, f, where the asymmetry is seen to change from dawn to dusk at lower energies under a northward IMF. This result is also contrary to the predictions of the gradient drift entry mechanism proposed by Olson and Pfitzer (1985). This mechanism predicts an increase in the occurrence of $\mathrm{He}^{2+}$ on the dawn flank with increasing ion energy.

\section{Summary and conclusions}

Figures $6 \mathrm{a}, \mathrm{b}$ show that for average energies less than $6.9 \mathrm{keV}$ the $\mathrm{He}^{2+}$ distribution is concentrated in the cusp near local noon and centred at $\Lambda \sim 79^{\circ}$ for southward IMF $\left(B_{Z}<0\right)$ and at $\Lambda \sim 81^{\circ}$ for northward IMF $\left(B_{Z}>0\right)$. This shows that, on average, the cusp shifts poleward for northward IMF and equatorward for southward IMF. This shift in the average location of the cusp is due to the fact that the dayside closed flux is less eroded by low-latitude reconnection when the IMF points northward.

At higher energies, $\mathrm{He}^{2+}$ ions in the magnetosphere, between $1.8-21.4 \mathrm{keV}$, are observed in the cusp around local noon and in the LLBL/auroral oval at all local times, as shown by the occurrence probabilities in Fig. 1. An asymmetry about the noon-midnight meridian shows that $\mathrm{He}^{2+}$ ions favour the dawn flank of the LLBL/auroral oval at these energies. This asymmetry has one or more of three potential causes: the Svalgaard-Mansurov effect; Fermi acceleration of $\mathrm{He}^{2+}$ at the bow shock; and convecting drift shells. The sense of the asymmetry introduced by the SvalgaardMansurov effect is dependent upon the IMF $B_{Y}$ direction (see Fig. 2) and, as shown by Figs. 3a, b, the asymmetry remains on the dawn flank for both IMF $B_{Y}>0$ and $B_{Y}<0$. Therefore, this effect is not responsible for the observed asymmetry. The asymmetry in the Fermi acceleration of $\mathrm{He}^{2+}$ ions at the bow shock depends on IMF $B_{X} / B_{Y}$ ratio. For a Parker spiral IMF, $B_{X} / B_{Y}<0$ (Fig. 4a), an asymmetry is expected on the dawn flank and vice-versa for ortho-Parker spiral IMF, $B_{X} / B_{Y}>0$ (Fig. $4 \mathrm{~b}$ ). Figures $5 \mathrm{a}$, b show the asymmetry persisting on the dawn flank. Therefore, Fermi acceleration at the bow shock does not explain this asymmetry either. To our knowledge, these are the only two asymmetries affecting ion entry into the magnetosphere and thus, from this, we conclude that the asymmetry is not formed by direct entry of $\mathrm{He}^{2+}$ ions from the solar wind onto open field lines.

This leaves ion drift shells on closed field lines convecting Earthward from the tail $X$-line. Figure 6 shows occurrence probabilities sorted by IMF $B_{Z}$ and ion energy. For energies ranging from 7.7 to $21.4 \mathrm{keV}$, occurrence probabilities are distributed from $68^{\circ}$ to $75^{\circ}$ invariant latitude at all local times. As shown in Figs. 6c, d, there is still a slight bias for the dawn flank for IMF $B_{Z}>0$ and a strong bias for IMF $B_{Z}<0$. For energies between 24.3 and $50.3 \mathrm{keV}$, the asymmetry changes from the dawn to dusk flank for both IMF orientations, (see Figs. 6e, f). The change to the dusk flank occurs at lower energies for IMF $B_{Z}>0$ as the convection electric field in the tail is weaker due to the reduction in sub-solar reconnection and global convection associated with northward IMF. The weaker electric field, in turn, results in a weaker $\boldsymbol{E} \times \boldsymbol{B}$ drift to the dawn flank to oppose the gradient $B$ and curvature drifts to the dusk flank. For southward IMF, the stronger convection electric field means $\mathrm{He}^{2+}$ ions need proportionally higher energies to get to the dusk flank. This evidence strongly suggests that drift shells on closed field lines are responsible for a dawn asymmetry in the occurrence probability of $\mathrm{He}^{2+}$ ions between 1.8 and $21.4 \mathrm{keV}$ in the magnetosphere.

If we compare our result with the Newell and Meng (1992) mapping of dayside ionosphere to the magnetosphere according to precipitation characteristics with data from the DMSP spacecraft, we find a good agreement with our survey from higher altitudes. They identify the cusp near local noon at $\Lambda \sim 78^{\circ}$, compared to our survey which locates it at $\Lambda \sim$ $79^{\circ}$. The distribution shown here in Figs. 6e, f map to the Boundary Plasma Sheet (BPS) and Central Plasma Sheet (CPS) in their survey. They show the BPS occupying the dawn and dusk flanks of the magnetosphere at $\Lambda<78^{\circ}$. The CPS is shown at all local times between 0600 and 1800 MLT with a high occurrence around 1000 MLT at $\Lambda<75^{\circ}$. The 
preference shown by the CPS ions for the dawn flank could well be caused by the drift orbits discussed here. Newell and Meng find the LLBL to be centred near 1030 MLT and range from 0600 to 1600 MLT and at invariant latitudes between $\Lambda \sim 75^{\circ}$ and $\Lambda \sim 80^{\circ}$. This covers the region where $\mathrm{He}^{2+}$ ions are found in Figs. 6a-d, but we do not see any major distinction between this LLBL location and the auroral oval in general in Fig. 6, and some of it extends into the region that we have characterised as cusp.

This result illustrates how magnetic reconnection and the associated global convection cycle is able to transport solar wind ions accessing the magnetosphere on open field lines and then subsequently circulate and energise them on closed field lines. In particular, it demonstrates that the distribution of $\mathrm{He}^{2+}$ ions with energies greater than $\sim 10 \mathrm{keV}$ at high invariant latitudes is dominated by ions drifting sunward in the magnetotail and that dawn-dusk asymmetry results from the combined effect of the convection electric field and gradient/curvature drifts.

Acknowledgements. This work was funded by PPARC. We thank the many individuals who have made the CAMMICE program (PI T. A. Fritz) a success. We have benefited from use of WIND MFI and SWE experiments (PIs R. P. Lepping and K. W. Ogilvie) supplied by NSSDC CDAWeb.

Topical Editor G. Chanteur thanks B. Wilken and H. E. Spence for their help in evaluating this paper.

\section{References}

Acuña, M. H., Ogilvie, K. W., Baker, D. N., Curtis, S. A., Fairfield, D. H., and Mish, W. H., The global geospace science program and its investigations, Space Sci. Rev., 71, 5, 1995.

Blandford, R. and Eichler, D., Particle acceleration at an astrophysical shock: A theory of cosmic ray origin, Phys. Reports, 154, 1, 1987.

Burch, J. L., Rate of erosion of dayside magnetic flux based on a quantitative study of polar cusp latitude on the interplanetary magnetic field, Radio Sci., 8, 955-961, 1973.

Chandler, M. O., Fuselier, S. A., Lockwood, M., and Moore, T. E., Evidence of component magnetic merging equatorward of the cusp, J. Geophys. Res., 104, 22623-22633, 1999.

Chen, J., Fritz, T. A., Sheldon, R. B., Spence, H. E., Spjeldvik, W. N., Fennell, J. F., Livi, S., Russell, C. T., Pickett, J. S., and Gurnett, D. A., Cusp energetic particle events: Implications for a major acceleration region of the magnetopshere, J. Geophys. Res., 103, A1, 69-78, 1998.

De Forest, S. A. and McIlwain, C. E., Plasma clouds in the magnetosphere, J. Geophys. Res., 76, 3587-6611, 1971.

Friis-Christensen, E., Kamide, Y., Richmond, A. D., and Matsushita, S., Interplanetary magnetic field control of high-latitude electric fields and currents determined from Greenland magnetometer data, J. Geophys. Res., 90, 1325, 1985.

Fuselier, S. A. and Schmidt, W. K. H., $\mathrm{H}^{+}$and $\mathrm{He}^{2+}$ heating at Earth's bow shock, , J. Geophys. Res., 99, 11539, 1994.

Fuselier, S. A. and Schmidt, W. K. H., Solar wind $\mathrm{He}^{2+}$ ring-beam distributions downstream from Earth's bow shock, J. Geophys. Res., 102, 11273, 1997.

Fuselier, S. A., Shelley, E. G., Peterson, W. K., and Lennartsson, O. W., Solar wind $\mathrm{He}^{2+}$ and $\mathrm{H}^{+}$distributions in the cusp for south- ward IMF, in Polar cap boundary phenomena, Eds. J. Moen, A. Egeland and M. Lockwood, pp 63-72, Kluwer, The Netherlands, 1998.

Gosling, J. T., Thomsen, M. F., Bame, S. J., Elphic, R. C., and Russell, C. T., Plasma flow reversals at the dayside magnetopause and the origin of asymmetric polar cap convection, J. Geophys. Res., 95, 8073-8084, 1990.

Grande, M., Fennell, J., Livi, S., Kellett, B., Perry, C., Anderson, P., Roeder, J., Spence, H., Fritz, T., and Wilken, B., First polar and 1995-034 observations of the midaltitude cusp during a persistent northward IMF condition, Geophys. Res. Lett., 24, 1475-1478, 1997.

Greenwald, R. A., Baker, K., Ruohoniemi, J. M., Dudeney, J. R., Pinnock, M., Mattinand, N., and Leonard, J. M., Simultaneous conjugate observations of dynamic variations in high-latitude dayside convection due to changes in IMF $B_{Y}$, J. Geophys. Res., 95, 8057, 1990.

Hapgood, M., Tulunay, A., Lockwood, M., Bowe, G., and Willis, D. M., Variability of the interplanetary medium at 1 A.U. over 24 years: 1963-1986, Planet. Space Sci., 39, 411-423, 1991.

Heelis, R. A., The effects of interplanetary magnetic field orientation on dayside high-latitude convection, J. Geophys. Res., 89, 2873, 1984.

Horwitz, J. L. and Akasofu, S.-I., The response of the dayside aurora to sharp northward and southward transitions of the interplanetary magnetic field and to magnetospheric substorms, J. Geophys. Res., 82, 2723-2734, 1977.

Lepping R. P., Acuña, M. H., Burlaga, L. F., Farrell, W. M., Slavin, J. A., Schatten, K. H., Mariani, F., Ness, N. F., Neubauer, F. M., Whang, Y. C., Byrnes, J. B., Kennon, R. S., Panetta, P. V., Scheifele, J., Worley, E. M., The WIND magnetic field investigation, Space Sci. Rev., 71, 207-229, 1995.

Newell, P. T, Meng, C.-I., Sibeck, D. G., and Lepping, R. P., Some low-altitude cusp dependencies on the interplanetary magnetic field, J. Geophys. Res., 94, 8921-8927, 1989.

Newell, P. T. and Meng, C.-I., Mapping the dayside ionosphere to the magnetosphere according to particle precipitation characteristics, Geophys. Res. Lett., 19, 609-612, 1992.

Ogilvie, K. W., Chornay, D. J., Fritzenreiter, R. J., Hunsaker, F., Keller, J., Lobell, J., Miller, G., Scudder, J. D., Sittler, E. C., Torbert, R. B., Bodet, D., Needell, G., Lazarus, A. J., Steinberg, J. T., and Tappan, J. H., SWE, A comprehensive plasma instrument for the WIND spacecraft, Space Sci. Rev., 71, 55-77, 1995.

Olson, W. P. and Pfitzer, K. A., Magnetospheric responses to the gradient drift entry of solar wind plasma, J. Geophys. Res., 90, 10823-10833, 1985.

Onsager, T. G., Thomsen, M. F., Elphic, R. C., and Gosling, J. T., Model of electron and ion distributions in the plasma sheet boundary layer, J. Geophys. Res., 96, 20999-21011, 1991.

Onsager, T. G., Scudder, J. D., Lockwood, M., and Russell, C. T., Reconnection at the high-latitude magnetopause during northward IMF conditions, J. Geophys. Res., submitted, 2000.

Perry, C. H., Grande, M., Zurbuchen, T. H., Hefti, S., Gloeckler, G., Fennell, J. F., Wilken, B., and Fritz, T., Use of Fe charge state changes as a tracer for solar wind entry to the magnetosphere, Geophys. Res. Lett., 27, 2441-2444, 2000.

Sandholt, P. E., Farrugia, C. J., Moen, J., and Cowley, S. W. H., Dayside auroral configurations: responses to southward and northward rotations of the interplanetary magnetic field, J. Geophys. Res., 103, 20279-20296, 1998.

Stubbs, T. J., Cargill, P. J., Grande, M., Kellett, B., Lockwood, M., and Perry, C., Observations of the northern polar cusp with 
T. J. Stubbs et al.: Dawn-dusk asymmetry in particles of solar wind origin within the magnetosphere

the POLAR spacecraft, Multiscale/multipoint plasma measurements, ESA SP-449, 2000.

Wilken, B., Weiß, W., Hall, D., Grande, M., Søraas, F., and Fennell,
J. F., Magnetospheric ion composition spectrometer onboard the CRRES spacecraft, Journal Spacecraft and Rockets, 29, 585591, 1992. 